\title{
EDITORIAL
}

\section{Clinical examination: a trigger but not a substitute for hemodynamic evaluation}

\author{
Daniel De Backer ${ }^{1 *}$ (10 and Antoine Vieillard-Baron ${ }^{2,3}$
}

(c) 2019 Springer-Verlag GmbH Germany, part of Springer Nature

Clinical examination is an essential part of the assessment of the critically ill patient. Among the different signs to look at, signs of tissue hypoperfusion and hemodynamic instability are particularly relevant. Several clinical signs can be used to detect tissue hypoperfusion. As an example, cerebral impairment, oliguria, capillary refill time, and mottling score are used in shock assessment [1]. Biological signs such as lactate have gained popularity, helping the clinician to suspect anaerobic metabolism, a potential consequence of severe hypoperfusion. Many of these signs are associated with outcome [2] and some may even be used to trigger resuscitation procedures [3].

While it is crucial to detect tissue hypoperfusion and its consequences, it is even more crucial to understand the cause of tissue hypoperfusion, whether cardiac output is sufficient, and what the cause of impaired cardiac output is. An important question is thus whether clinical and/or biological signs can predict cardiac index (CI). Tachycardia and hypotension mainly reflect the impaired cardiovascular status but it is obvious that neither tachycardia nor hypotension can reliably predict CI.

In this issue, Hiemstra et al. [4] prospectively evaluated in 783 critically ill patients whether 19 clinical and biological signs (among which heart rate, blood pressure, respiratory rate, cardiac and lung auscultation, mental state, urine output, and various signs of skin perfusion), evaluated in a protocolized manner, could predict CI. The authors first used linear regression to evaluate variables potentially associated with CI. Next they conducted

*Correspondence: ddebacke@ulb.ac.be

${ }^{1}$ Department of Intensive Care, CHIREC Hospitals, Université Libre de Bruxelles, Brussels, Belgium

Full author information is available at the end of the article multivariate analyses to determine which combined clinical signs were identified with low or high CI.

In this single-center study, CI was measured by critical care echocardiography, using a transthoracic route, by experts in the field. The first interesting result is that among the 1212 eligible patients (unplanned ICU admission, expected to stay for at least $24 \mathrm{~h}$ ), echocardiography could not be performed for technical reasons (chest drains, proning, wounds, etc.) in 80 patients $(6.6 \%)$ and, when performed, was of insufficient quality to obtain CI in 292 other patients (24\%). In trauma patients, factors such as a weight gain greater than $10 \%$, a PEEP of at least $15 \mathrm{cmH}_{2} \mathrm{O}$, and chest tubes may contribute to the high failure rate (38\%) of transthoracic echocardiography imaging [5]. Curiously, Hiemstra et al. [4] reported an unexpected similar failure rate in this study that included mostly medical patients. Even more surprising, Hiemstra et al. [6] previously mentioned in their preliminary report of the first 704 included patients that echocardiography was judged by experts to be of sufficient quality in 632 patients (90\%), a much more usual failure rate. The second interesting finding is that $\mathrm{CI}$ was frequently low in this unselected population of ICU patients. A low CI (below $2.2 \mathrm{l} / \mathrm{min} / \mathrm{m}^{2}$ ) was observed in $36 \%$ of the patients and $49 \%$ of the patients had a CI below $2.5 \mathrm{l} / \mathrm{min} / \mathrm{m}^{2}$. Identifying these patients who potentially may benefit from hemodynamically targeted interventions is thus relevant.

Twelve clinical signs differed between patients with low and high CI. Seven signs reflecting impaired respiration, central circulation and skin perfusion were independently associated with CI. Five clinical signs (atrial fibrillation, systolic blood pressure below $90 \mathrm{mmHg}$, altered mental state, capillary refill time greater than $4.5 \mathrm{~s}$ at the sternum, and knee skin mottling) had a high specificity to detect a low CI. Interestingly, mean arterial

\section{Springer}




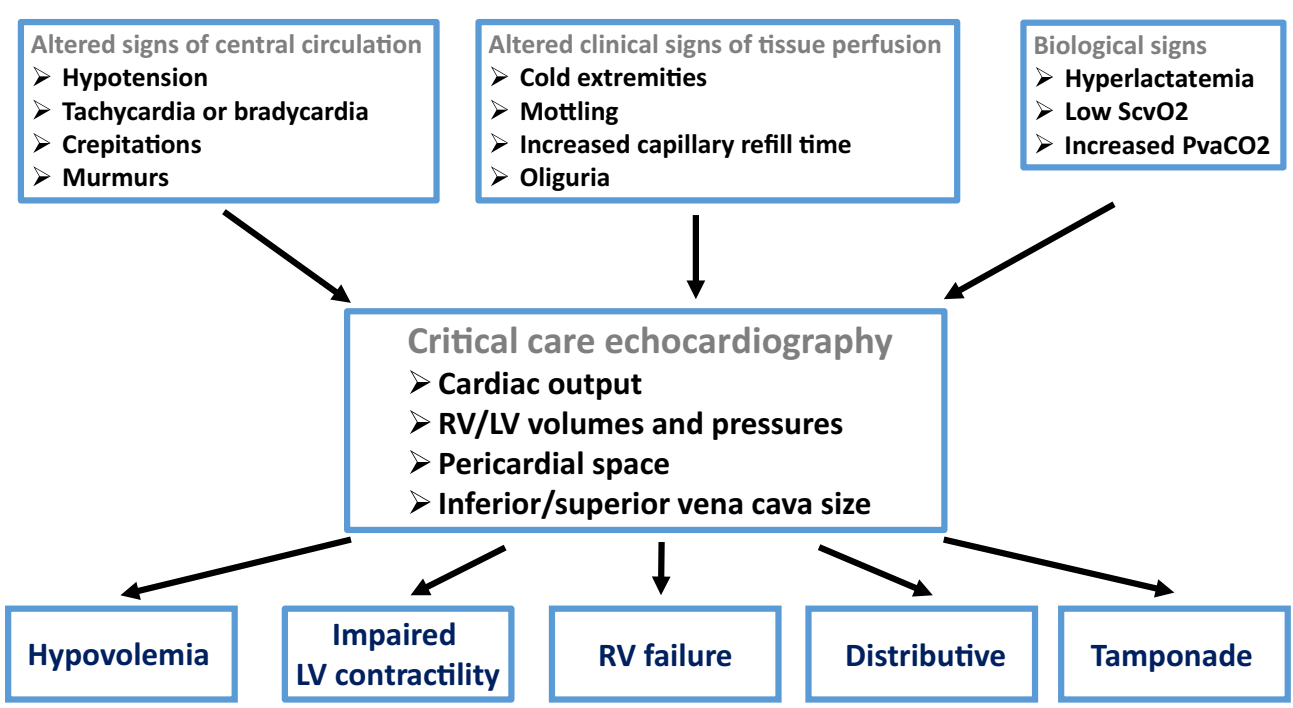

Fig. 1 Proposed diagnostic workflow in patients with signs of altered hemodynamics. ScvO2 denotes central venous oxygen saturation, PvaCO2 veno-arterial difference in PCO2, LV left ventricle, RV right ventricle

pressure, central venous pressure, and urine output did not differ between the various $\mathrm{CI}$ level groups.

Unfortunately, the diagnostic accuracy was insufficient to identify low $\mathrm{CI}$ on the basis of these clinical signs. Even though most patients had altered signs of central hemodynamics and of tissue perfusion, the sensitivity of these signs was poor. Indeed, among the 368 patients with cardiac index below $2.2 \mathrm{~L} / \mathrm{min} / \mathrm{m}^{2}, 34$ had a systolic blood pressure below $90 \mathrm{mmHg}$ and 52 a mean arterial pressure below $65 \mathrm{mmHg}, 177$ had cold extremities by subjective assessment, 38 had prolonged capillary refill time at the sternum, and 74 had severe skin mottling. The five highly specific signs mentioned above were present in only $10 \%$ of the patients with low CI. In addition to the very low the positive predictive value (ranging between $30 \%$ and $50 \%$ for most signs), the negative predictive value was only slightly better, ranging between $60 \%$ and $75 \%$. This low positive predictive value is an important result as it is more representative of the physician's way of thinking in trying to detect a low $\mathrm{CI}$ rather than specificity. Indeed, it defines the probability that a low $\mathrm{CI}$ is present given the observed clinical signs. Furthermore, differences in the different variables between low and high CI were sometimes relatively minimal. For example, only $1 \mathrm{~s}$ in capillary refill time separated low and high CI [3.0 (3.0-4.0) s] in low CI patients and 2.0 (2.0-3.0) $\mathrm{s}$ in high CI patients.

While Hiemstra et al's results were somewhat expected from studies using either the pulmonary artery catheter [7] or transpulmonary thermodilution [8], the authors have to be congratulated as their rigorous analysis definitively gives us key information about the value of usual clinical signs.
What should we do next with these signs? We should not throw the baby with the bathwater. These signs are very useful to identify that there was a hemodynamic/ perfusion issue. The included patients were very severely ill, half of them receiving norepinephrine, and very few patients (5\%) had all clinical signs normal. Three quarters of the patients had abnormal skin perfusion signs, $68 \%$ abnormal signs of renal perfusion and one quarter signs of abnormal cerebral perfusion. Accordingly, these signs performed relatively well in indicating that there was a problem, but did not help so much in indicating what the source of the problem was.

We suggest, in accordance with the ESICM consensus report on hemodynamic management of shock [9], to use clinical sings to identify patients with potential hemodynamic issues, then to rapidly perform critical care echocardiography [10] in order to identify the type of shock and the source, if any, of the hemodynamic problem (Fig. 1).

In conclusion, clinical signs are important warning signals indicating that something is going wrong but these cannot reliably indicate whether cardiac index is low or high, nor indicate the source of the hemodynamic alteration. Physicians should not base their decisions/management solely on these clinical signs. The presence of altered clinical signs should prompt physicians to perform additional hemodynamic evaluations and in particular echocardiography.

\section{Author details}

${ }_{1}^{1}$ Department of Intensive Care, CHIREC Hospitals, Université Libre de Bruxelles, Brussels, Belgium. ${ }^{2}$ Medical-surgical Intensive Care Unit, Ambroise Paré 
University Hospital, APHP, Boulogne-Billancourt, France. ${ }^{3}$ Université Versailles Saint Quentin, INSERM UMR1018, Team Kidney and Heart, CESP, Villejuif, France.

\section{Compliance with ethical standards}

\section{Conflicts of interest}

Daniel De Backer: Consultant to and material for studies by Edwards Lifesciences. Antoine Vieillard-Baron: Received grant from GSK for conducting clinical research and was member of the scientific advisory board for the study.

\section{Ethical approval}

An approval by an ethics committee was not applicable.

\section{Publisher's Note}

Springer Nature remains neutral with regard to jurisdictional claims in published maps and institutional affiliations.

Received: 15 January 2019 Accepted: 17 January 2019

Published online: 24 January 2019

\section{References}

1. Vincent JL, De Backer D (2013) Circulatory shock. N Engl J Med 369:1726-1734

2. Ait-Oufella H, Bakker J (2016) Understanding clinical signs of poor tissue perfusion during septic shock. Intensive Care Med 42:2070-2072
3. Hernandez G, Cavalcanti AB, Ospina-Tascon G, Zampieri FG, Dubin A, Hurtado FJ et al (2018) Early goal-directed therapy using a physiological holistic view: the ANDROMEDA-SHOCK_a randomized controlled trial. Ann Intensive Care 8:52

4. Hiemstra B, Koster G, Wiersema R, Hummel Y, van der Harst P, Snieder H et al (2019) The diagnostic accuracy of clinical examination for estimating cardiac output in critically ill patients: the Simple Intensive Care Studies-I. Intensive Care Med. https://doi.org/10.1007/s00134-019-05527-y

5. Cook CH, Praba AC, Beery PR, Martin LC (2002) Transthoracic echocardiography is not cost-effective in critically ill surgical patients. J Trauma 52:280-284

6. Hiemstra B, Eck RJ, Koster G, Wetterslev J, Perner A, Pettila V et al (2017) Clinical examination, critical care ultrasonography and outcomes in the critically ill: cohort profile of the Simple Intensive Care Studies-I. BMJ Open 7:e017170

7. Dawson NV, Connors AF, SperoffT, Kemka A, Shaw P, Arkes HR (1993) Hemodynamic assessment in managing the critically ill: is physician confidence warranted? Med Decis Mak 13:258-266

8. Perel A, Saugel B, Teboul JL, Malbrain ML, Belda FJ, Fernandez-Mondejar E et al (2016) The effects of advanced monitoring on hemodynamic management in critically ill patients: a pre and post questionnaire study. J Clin Monit Comput 30:511-518

9. Cecconi M, De Backer D, Antonelli M, Beale RJ, Bakker J, Hofer C et al (2014) Consensus on circulatory shock and hemodynamic monitoring. Task Force of the European Society of Intensive Care Medicine. Intensive Care Med 40:1795-1815

10. Expert round table on echocardiography in ICU (2014) International consensus statement on training standards for advanced critical care echocardiography. Intensive Care Med 2014(40):654-666 